\title{
Quantitative and Transverse Changes in the Position of Palatal Rugae after Palatal Expansion
}

Alka Banker*, Ketukumar D.Patel, Ipsit Trivedi, Riddhi Patel and Jayasankar P.Pillai

Goenka Research Institute of Dental Science, Gujarat, India

*Corresponding author: Alka Banker, Goenka Research Institute of Dental Science, Gujarat, India, Tel: 919824002666; E-mail: bankeralka@yahoo.com Rec date: July 09, 2016; Acc date: July 19, 2016; Pub date: July 26, 2016

Copyright: (c) 2016 Banker A et al. This is an open-access article distributed under the terms of the Creative Commons Attribution License, which permits unrestricted use, distribution, and reproduction in any medium, provided the original author and source are credited.

\begin{abstract}
Background: Palatal rugae are irregular and non-identical mucosal elevations seen on anterior third of palate. They are arranged in transverse direction on either side of Median Palatine Raphe [MPR]. Though they are the most stable anatomical structures in the oral cavity, certain conditions like orthodontic treatment contribute to some amount of variations in their pattern qualitatively. During literature review, we found scarcity of articles related to quantitative changes in rugae patterns, before and after palatal expansion. The aim of this study was to compare the number of rugae and its positional changes using before and after Semi Rapid Maxillary Expansion (SRME) study models.
\end{abstract}

Materials and Methods: Seventy nine pairs of pre and post expansion casts showing finer details of palatal rugae from 37 males and 42 females in the age group of 10 to 12 years were selected for this study. The number of rugae and the distance between the median points of the first and the last two rugae on either side of MPR were noted. The data collected were statistically analyzed using SPSS program

Results: Descriptive statistics mean and standard deviation were performed for study group before and after treatment at T0 and T1. There was no statistically significant change in number of rugae in the right side but significant differences were noted in the distance between medial points of first two rugae and distance between last two rugae. There was statistically no significant change in any of the variables when the data from male and female samples were compared.

Conclusion: During maxillary expansion, there is stability of palatal rugae with respect to its number but not with respect to its position. There is significantly more separation of the medial ends of the opposing rugae on either side of MPR in the anterior region than on the posterior.

Keywords: Rugae; Semi rapid maxillary expansion; Medial ends; Mid palatal raphe

\section{Introduction}

Palatal rugae are considered to be stable anatomical landmarks; however certain conditions like thumb sucking, trauma and orthodontic treatments contribute to some changes in their patterns [1]. During orthodontic treatments, palatal rugae are used a reference points for the quantification of tooth migration [2]. The palatal rugae are unique oral topographical structures to an individual, like his or her finger printsand this characteristic pattern remains unchanged throughout life [3]. However some authors have reported changes in the mean rugae count with age [4]. The palatal rugae are classified and studied qualitatively based on parameters like length, shape, direction and unification and quantitatively based on the number of rugae present on either side of mid palatine raphe. The number of rugae may or may not be the same on either side of MPR. The length of an individual ruga is usually measured from its starting point near the mid palatine raphe to its end point transversely. According to Van der Linden the length of anterior rugae remains stable after 10 years of age [5]. But other investigators have revealed changes in length of rugae with age $[2,4]$. Some external factors like trauma, finger sucking, dentures, extractions of teeth and orthodontic treatments also have contributed to changes in rugae pattern and number $[6,7]$.

Malocclusion in early development, especially transverse discrepancies lead to facial asymmetry and subsequent functional and aesthetic problems. Maxillary expansion is the solution for correcting such transverse discrepancies where the mid palatal suture is widened using expansion plates with screws. During such expansion of the palate, metric changes occur in rugae. An increase in the distance between the medial points of the opposing rugae has been noted. More separation of the MPR is anticipated in the anterior part than on the posterior region hence the distance between the medial points of the right and left first rugae should increase more than those of the last rugae on either side. The aim of this study was to identify the quantitative and metric changes in the first and last pair of rugae on either side of MPR following maxillary expansion using pre and post expansion casts.

\section{Materials and Method}

Seventy nine pairs of pre and post treatment casts of 37 males and 42 female patients attending the specialty dental clinic were selected from the archives for this study. The study subjects were the patients treated with semi rapid palatal expansion brought about by a removable plate, with an average time of $3.5 \pm 0.5$ months between pre 
and post treatment. The outline of the palatal rugae was highlighted using a micro tip pencil. The number of rugae on the right and left side of mid palatine raphe was noted down and the distance between the medial ends of the anterior most and the posterior most rugae on either side of MPR were measured in both pre and post treatment casts using a divider and scale. The data thus obtained were entered in an excel sheet and descriptive statistical analysis was performed. Mann Whitney $U$ test was performed to compare the mean difference between male and female values and Wilcoxon test for significance was performed to compare mean difference between before and after treatment values.

\section{Result}

Pre and post expansion casts of 39 males and 40 female subjects were included in the study. A total of 620 rugae was observed in the before expansion casts with a mean count of 7.84. In the post expansion casts, a total of 616 rugae were observed with a mean count of 7.79. There was a net difference of 4 rugae count in the before and after expansion casts. Quantitatively the rugae count in the left side of the MPR was more than those on the right side in both before and after expansion casts. It was observed that there was an increase of one ruga on the right side and a decrease of 5 rugae count in the left side when the before and after expansion casts were compared. However there was no significant difference in the total rugae count when the pre and post expansions casts were compared. There was also no significant difference between the numbers of rugae on the right and left side of MPR in pre and post treatment casts were compared. The mean distance between the medial ends of the right and left first rugae was $2.62 \mathrm{~mm}$ and $5.29 \mathrm{~mm}$ for the last two rugae in the pre-treatment models. There was significant difference in those inter ruga distance when the pre and post expansion casts were compared (Table 1). The mean changes in the variables before and after treatment between males and females are described in Table 2. It was observed that there is no statistical significance in any of the variables.

\begin{tabular}{|c|c|c|c|c|c|c|}
\hline \multirow{2}{*}{ Variables } & \multicolumn{2}{|l|}{ Pre } & \multicolumn{2}{|l|}{ Post } & \multirow[t]{2}{*}{$\begin{array}{l}P \\
\text { value }\end{array}$} & \multirow[t]{2}{*}{$\begin{array}{l}\text { Significanc } \\
\mathrm{e}\end{array}$} \\
\hline & Mean & SD & Mean & SD & & \\
\hline No. of Rugae_Rt. & 3.75 & 0.95 & 3.77 & 0.96 & 0.868 & NS \\
\hline No. of Rugae_Lt. & 4.09 & 0.92 & 4.03 & 0.89 & 0.66 & NS \\
\hline $\begin{array}{l}\text { Dist. between 1st two } \\
\text { Rugae (mm.) }\end{array}$ & 2.62 & 1.01 & 3.46 & 1.22 & 0 & $\mathrm{~s}$ \\
\hline $\begin{array}{l}\text { Dist. between last two } \\
\text { Rugae }(\mathrm{mm} \text {.) }\end{array}$ & 5.29 & 2.34 & 6.06 & 2.46 & 0 & $\mathrm{~s}$ \\
\hline Total Rugae count & 7.87 & 1.66 & 7.74 & 1.62 & 0.14 & NS \\
\hline \multicolumn{7}{|c|}{$\mathrm{S}=$ Significance, NS=Non Significance, $p \leq 0.05$} \\
\hline
\end{tabular}

Table 1: Quantitative and metric changes in rugae in before and after expansion casts

\begin{tabular}{|c|c|c|c|c|c|c|c|c|c|c|}
\hline \multirow{3}{*}{ Variables } & \multicolumn{4}{|c|}{ Pre Treatment } & \multicolumn{4}{|c|}{ Post treatment } & \multirow{4}{*}{$\begin{array}{l}p \text { value } \\
0.129\end{array}$} & \multirow{4}{*}{$\begin{array}{l}\text { Significance } \\
\text { NS }\end{array}$} \\
\hline & \multicolumn{2}{|l|}{ Male } & \multicolumn{2}{|c|}{ Female } & \multicolumn{2}{|l|}{ Male } & \multicolumn{2}{|c|}{ Female } & & \\
\hline & Mean & SD & Mean & SD & Mean & SD & Mean & SD & & \\
\hline No. of Rugae(Rt.) & 3.66 & 0.83 & 3.85 & 1.05 & 3.76 & 0.95 & 3.77 & 0.97 & & \\
\hline No. of Ruga(Lt.) & 4.1 & 1.11 & 4.12 & 0.85 & 3.97 & 0.95 & 3.97 & 0.8 & 0.831 & NS \\
\hline Dist. between 1st two Rugae (mm.) & 2.35 & 0.93 & 2.88 & 1.02 & 3.32 & 1.22 & 3.6 & 1.23 & 0.533 & NS \\
\hline Dist. between last two Rugae (mm.) & 5.05 & 2.28 & 5.52 & 2.4 & 5.79 & 2.35 & 6.32 & 2.57 & 0.452 & NS \\
\hline Total Rugae count & 7.76 & 1.69 & 7.97 & 1.65 & 7.74 & 1.63 & 7.75 & 1.62 & 0.122 & NS \\
\hline
\end{tabular}

Table 2: The mean values of the variables in pre and post treatment models of male and female samples

\section{Discussion}

Palatal rugae are considered to be the most stable anatomic structures in the oral cavity. However there is more likelihood for the changes in the shape, number and position of rugae following orthodontic treatment as there is significant increase in the arch perimeter in such cases. Such changes in the rugae patterns were studied in the past by several investigators [8,9]. The rugae landmarks are also use reliably as reference points for cephalometric superimposition when assessing the antero-posterior tooth movement during orthodontic treatments [10,11]. Metric changes in the transverse plane between the medial ends of bilateral opposing rugae were observed in rapid maxillary expansion cases. Damstra et al. have demonstrated more marked transverse changes in the third rugae when compared to the second and first rugae. Thus they concluded that third rugae cannot be considered a stable reference landmark for dental cast analysis [12]. An Indian study revealed significant difference in the distance between the lateral points of $1^{\text {st }}$ rugae when the pre and post palatal expansion casts were compared. They concluded that the most reliable stable points were the medial and lateral $3^{\text {rd }}$ rugae points. A similar study done on Egyptian patients [13] suggested that the lateral $3^{\text {rd }}$ rugae points were the most reliable points for superimposition while comparing the pre and post orthodontically treated casts. In our study, we measured the transverse distance between the medial ends of the first two and the last two rugae on either side of Mid Palatal Raphe [MPR] or found significant difference in the measurements in the pre-treatment and post treatment casts. Changes in respect to shape of palatal rugae on both sides were observed in palatal expansion and in extraction cases in a study done 
by Deepak et al. [14]. They observed an increase in the length of rugae on right side in palatal expansion cases. A similar study by Bansode et al. showed changes in the length of palatal rugae during palatal expansion [15]. In our study, we did not consider the length of rugae as a parameter; instead we included the number and the transverse distance between the medial ends of first two and the last two rugae. The metric and the positional stability of $3^{\text {rd }}$ rugae during orthodontic treatment has been documented in several studies [2,5,10-12]. Kratzsch et al., in their series of investigations on palatal rugae have compared the distances between lateral palatal rugae points and to specific landmarks in maxilla. They indicated that changes occurred in the anterior palate during various stages of growth and orthodontic treatment [16]. In this study we measured the distance between two pairs of rugae - first and last pair, post SRME treatment and found a significant change of $0.84 \mathrm{~mm}$ increase in distance between the first pair and $1.77 \mathrm{~mm}$ increase between the last pair. Skeletal expansion of the maxilla had a triangular pattern with a wider base in the anterior region. This is because the opening of mid palatal suture is greater in the anterior region than the posterior region. Other studies also showed similar results $[17,18]$. Garrett et al. [19] in their study showed that $55 \%$ of total expansion was at 1 st pre molar level (P1), $45 \%$ at 2 nd Premolar level (P2), and 38\% at 1st Molar level (M1). During expansion there is rotation of maxillary components in the frontal and horizontal planes. This was proved by Krebs [20] using metallic implants in his study. The fulcrum of maxillary rotation during expansion is at the fronto-maxillary suture as well as at the pterygopalatine suture [21]. The increase in distance between the palatal rugae implies that there was a skeletal expansion at the maxillary suture, which is the main goal of expansion treatment. This study also found no change in the number of rugae post expansion treatment. There was also no change seen in the distances and number of rugae as compared between males and females (Figure 1).



Figure 1: Schematic rugae

\section{Conclusion}

- Quantitatively the palatal rugae may be considered as a stable anatomical landmark.

- The inter ruga distance at the first and last pairs of rugae on either side of MPR differ significantly when pre and post treatment models were compared.
- Clinically the orthodontist can quantify the palatal separation using the inter-ruga distance.

\section{References}

1. Kapali S, Townsend G, Richards L, Parish T (1997) Palatal rugae patterns in Australian aborigines and Caucasians. Aust Dent J 42: 129-133.

2. Simmons JD, Moore RN, Erickson LC (1987) A longitudinal study of anteroposterior growth changes in the palatine rugae. J Dent Res 66: 1512-1515.

3. English WR, Robison SF, Summitt JB, Oesterle LJ, Brannon RB, et al. (1988) Individuality of human palatal rugae. J Forensic Sci 33: 718-726.

4. Hauser G, Daponte A, Roberts MJ (1989) Palatal rugae. J Anat 165: 237-249.

5. Van der Linden FP (1978) Changes in the position of posterior teeth in relation to ruga points. Am J Orthod 74: 142-161.

6. Peavy DC Jr, Kendrick GS (1967) The effects of tooth movement on the palatine rugae. J Prosthet Dent 18: 536-542.

7. Shukla D, Chowdhry A, Bablani D, Jain P, Thapar R (2011) Establishing the reliability of palatal rugae pattern in individual identification (following orthodontic treatment). J Forensic Odontostomatol 29: 20-29.

8. Bailey LT, Esmailnejad A, Almeida MA (1988) Stability of the palatal rugae as landmarks for analysis of dental cast in extraction and non extraction cases. J Forensic Sci 33: 718-726.

9. Almeida MA, Phillips C, Kula K, Tulloch C (1995) Stability of the palatal rugae as landmarks for analysis of dental casts. Angle Orthod 65: 43-48.

10. Hoggan BR, Sadowsky C (2001) The use of palatal rugae for the assessment of anteroposterior tooth movements. Am J Orthod Dentofacial Orthop 119: 482-488.

11. Janga I, Tanaka M (2009) A novel method for the assessment of threedimensional tooth movement during Orthodontic treatment. Angle Orthodont 79: 447-453.

12. Damstra J, Mistry D, Cruz C, Ren Y (2009) Antero-posterior and transverse changes in the positions of palatal rugae after rapid maxillary expansion. Eur J Orthod 31: 327-332.

13. Abdel-Aziz HM, Sabet NE (2001) Palatal rugae area: a landmark for analysis of pre- and post-orthodontically treated adult Egyptian patients. East Mediterr Health J 7: 60-66.

14. Deepak V, Malgaonkar NI, Shah NK, Nasser AS, Dagrus K, et al. (2014) Palatal rugae patterns in orthodontically treated cases, are they a reliable forensic marker? J Int Oral Health 6: 89-95.

15. Bansode SC, Kulkarni MM (2009) Importance of palatal rugae in individual identification. J Forensic Dent Sci 1: 77-81.

16. Kratzsch H, Opitz C (2000) Investigations on the palatal rugae pattern in cleft patients. Part II: Changes in the distances from the palatal rugae to maxillary points. J Orofac Orthop 61: 421-431.

17. Timms DJ (1999) The dawn of rapid maxillary expansion. Angle Orthod 69: 247-250.

18. D'Souza IM, Kiran Kumar HC, Shetty KS (2015) Dental arch changes associated with rapid maxillary expansion: A retrospective model analysis study. Contemp Clin Dent 6: 51-57.

19. Garrett BJ, Caruso JM, Rungcharassaeng K, Farrage JR, Kim JS, Taylor GD (2008) Skeletal effects to the maxilla after rapid maxillary expansion assessed with cone-beam computed tomography. Am J Orthod Dentofacial Orthop 134: 8-9.

20. Kerbs A (1958) Expansion of mid palatal sutures studied by means of metallic implants. European Orthodont Soc Rep 34: 163-171

21. Wertz R, Dreskin M (1977) Midpalatal suture opening: a normative study. Am J Orthod 71:367-381. 